\title{
L'évaluation de la dimension architecturale des établissements scolaires récents. Enquête sur le ressenti des collégiens
}

Assessment of the architectural dimension of recent schools. Survey on the feelings of high school students

Thibaut Hébert et Eric Dugas

\section{OpenEdition}

Journals

Édition électronique

URL : https://journals.openedition.org/trema/5460

DOI : $10.4000 /$ trema. 5460

ISSN : 2107-0997

Éditeur

Faculté d'Éducation de l'université de Montpellier

Référence électronique

Thibaut Hébert et Eric Dugas, «L'évaluation de la dimension architecturale des établissements scolaires récents. Enquête sur le ressenti des collégiens », Tréma [En ligne], 52 | 2019, mis en ligne le 01 septembre 2019, consulté le 16 janvier 2023. URL : http://journals.openedition.org/trema/5460 DOI : https://doi.org/10.4000/trema.5460

Ce document a été généré automatiquement le 16 janvier 2023.

Tous droits réservés 


\title{
L'évaluation de la dimension architecturale des établissements scolaires récents. Enquête sur le ressenti des collégiens
}

\author{
Assessment of the architectural dimension of recent schools. Survey on the \\ feelings of high school students
}

Thibaut Hébert et Eric Dugas

\section{Introduction}

1 Interprétée en termes de bien-être subjectif des individus (Layard, 2007), de niveau de vie (Jackson, 2002) ou de développement durable (cf. rapport Brundtland, 1987), la qualité de vie ne peut être dissociée du rapport de l'Homme à son environnement. Si cette expérience subjective tient en partie à «des valeurs, des perceptions et des aspirations de chacun » (Divay et al., 2004, p. 66), néanmoins, « une relation positive au lieu de vie est essentielle pour le bien-être de l'individu» (Moser, 2009, p. 236). Autrement dit, les lieux dans lesquels nous vivons, apprenons, travaillons, ne sont pas de simples décors (Moser, 2009) et la manière dont l'individu interagit avec son environnement conditionne sa qualité de vie. Cette relation s'opère à la fois avec les dimensions physiques et sociales de l'environnement car celui-ci « forme le cadre social dans lequel nous vivons » (Fischer, 2011, p. 14). Elle se produit sur plusieurs niveaux de l'environnement: micro, méso, macro et global (Moser, 2009). Ce concept étant protéiforme, l'environnement concerne le lieu de vie de l'individu, le bâti (Moser, 2009), ou encore l'espace aménagé dans nos sociétés (Fischer, 2011).

2 Ainsi, depuis une cinquantaine d'années, des chercheurs pointent les conséquences de l'action spatiale sur nos comportements (Jacobs, 1961 ; Newman, 1973) dans les espaces privatifs (logement, etc.), partagés (quartier, lieu de travail, hôpital, etc.), collectifs 
publics (villes, villages, etc.). Ils précisent les facteurs architecturaux d'influence négative (pollution, bruit, densité, absence de lumière et de couleur, espaces peu aménagés, manque d'esthétisme) afin d'infléchir les programmes de construction favorables au bien-être des individus. Par exemple, les travaux sur l'influence du bruit sur une activité (Guélaud et al., 1975), sur la qualité des relations avec autrui (Fischer, 2011) ou sur les enfants (Cohen et al., 1973; Moch, 1985) ont pu permettre des avancées significatives sur l'utilisation de matériaux renforçant le confort acoustique. La prise en compte des conséquences de l'entassement et de la densité sur les sentiments d'insécurité et de liberté (Stockdale, 1978) a également contribué à modifier l'approche des logements urbains. Comprendre l'impact de la dimension esthétique de l'environnement physique sur l'individu a certainement participé au renouvellement de l'habitat, des logements collectifs et des établissements publics dans nos villes et nos campagnes. En effet, le jugement esthétique d'un bâtiment influe sur « le bien-être et la fatigue (Maslow et Minz, 1956, in Broadbent, 1987), (...) l'identité et l'estime de soi des utilisateurs (Twigger-Ross et Uzzell, 1996; Uzzell, Pol et Badenes, 2002)»(Uzzel, Romice, 2003, p.51) et sur le développement des sentiments d'appartenance et d'insécurité (Newman, 1972).

3 Tous ces travaux ont donc permis d'identifier un certain nombre de "stresseurs de l'environnement» (Fischer, 2011). Par conséquent, il est désormais considéré que «l'environnement et les carences environnementales sont susceptibles d'être perçues comme une menace pour la qualité de vie » (Moser, 2009, p. 236).

\section{Du contexte urbain au contexte scolaire}

4 Au niveau méso-environnement, l'école, qui ne cesse de faire sa mue, n'échappe pas à ces avancées. Alors que la conception architecturale des établissements scolaires a évolué depuis l'avènement de la révolution industrielle du début du XIXe siècle (notamment Toulier, 1982; Châtelet, 1999, 2004), il semble que des réflexions s'engagent enfin pour concevoir des bâtiments répondant aux enjeux pour l'école de demain. Si les chercheurs en sciences de l'éducation ont pendant longtemps peu étudié l'architecture scolaire et ses effets, depuis quelques années, plusieurs publications ${ }^{1}$, colloques $^{2}$ ou projets innovants ${ }^{3}$ ont valorisé le sujet en analysant les bouleversements en cours (refondation de l'école, ère du numérique, problématiques de la sécurité et du climat scolaire, etc.) et en imaginant des espaces adaptés à ces mutations. Leurs travaux révèlent ainsi que le bâtiment scolaire et l'aménagement des espaces impactent le bienêtre des élèves (Derouet-Besson, 2007 ; Mazalto, 2013, 2015 ; Hébert et Dugas, 2017, etc.), leur engagement dans les apprentissages (Barker et Gump, 1964) et leur réussite éducative (Moch, 1985 ; Barett et al., 2015).

5 En voie de conséquences, afin d'agir sur les effets négatifs des «stresseurs de l'environnement " suscités (Hébert, 2019), il semblerait qu'une série d'actions soit engagée sur les caractéristiques physiques de l'espace scolaire par les architectes et les collectivités territoriales. Pour réduire la densité subjective, le sentiment d'entassement et maîtriser leurs effets sur l'agressivité et les comportements incivils (Lévy-Leboyer, 1980, Moscovici, 1988, Moser, 2009), les nouveaux collèges se veulent "de taille humaine " n'excédant pas 600 élèves et les lieux de circulation comme les couloirs sont élargis (Hébert, 2019). Pour limiter l'impact négatif du bruit sur les performances des élèves (Bronzaft et Mc Carthy, 1975), la mise en place de matériaux 
améliorant le confort phonique a pu être opérée (Hébert, 2019). « La démarcation entre les différents espaces (salles de classe, centre de documentation, espaces de vie scolaire, salles informatiques), devient peu à peu plus subtile (Dizerbo, 2017) et tend à rendre les établissements plus agréables et davantage sécurisants (Hébert, 2019). Des collèges, récemment construits, affichent des façades modernes, où de larges vitres cohabitent avec des bardages en bois. En somme, depuis une petite dizaine d'années, il semblerait que toute une série d'actions sur les caractéristiques physiques des établissements scolaires fasse consensus. Globalement, architectes, financeurs et chefs d'établissement (Hébert, 2018, 2019) semblent enfin considérer que les « caractéristiques, techniques et matérielles des locaux jouent un rôle incontestable sur l'ambiance de l'établissement " (Derouet Besson, 2007, p. 127). Cet engagement semble donner au premier abord un nouveau souffle aux établissements scolaires.

6 Pour autant tous ces actes sont-ils évalués? Et surtout, comment les usagers les perçoivent-ils? Sont-ils questionnés et légitimés? Plusieurs éléments pourraient laisser augurer un ressenti contrasté des acteurs de l'école, malgré l'émergence établissements neufs :

7 D'une part, certains redoutent que la sanctuarisation des établissements scolaires constatée depuis les années $2006^{4}$ fasse écho à l'émergence d'enclaves résidentielles valorisant l'entre-soi (Loudier et Vallet, 2010) et à une logique «d'enfermement et d'assujettissement» constatée notamment dans les centres commerciaux (Paquot, 2015, p. 25). D'autre part, des architectes et chefs d'établissement abordent le bâtiment scolaire comme un lieu défensif où la sécurité, considérée comme le levier central du bien-être des élèves, est abordée à travers une double dialectique ouverture / fermeture et liberté / coercition (Hébert, 2019). Autrement dit, les doubles parvis ou les grilles sont préconisés, et les caméras de surveillance sont encouragées. Ces perceptions viennent consolider les observations de Mazalto (2013) sur l'absence d'aménagements et d'espaces verts dans les cours de récréation. Enfin, des élèves ont déjà critiqué les qualités architecturales de leur bâtiment scolaire récemment construit. Pour exemple, le collège Miriam-Makeba, inauguré à la rentrée 2015 dans le nord de la France reçut un accueil très mitigé de la part des élèves ${ }^{5}$ :

«Les classes sont bien, mais juste il y a beaucoup de ciment. »

"C'est que du gris.»

«La façade n'est pas belle, c'est tout marron et noir. Avec des couleurs vives ou du

blanc, ça ferait mieux. Quand on rentre, avec ces barreaux à la porte, et les caméras

partout, on dirait que c'est la prison. »

8 Ceci interroge forcément sur les orientations actuelles des financeurs et architectes. Cela nous questionne d'autant plus que dans le cadre urbain, nous avons évoqué l'importance de la qualité environnementale (espaces aménagés, prise en compte des stresseurs, esthétisme).

9 À l'heure où les enquêtes révèlent une certaine désaffection des élèves français à l'égard de l'école (Debarbieux, 2011, Guimard et al., 2015), il paraît donc opportun de porter notre regard sur l'environnement physique scolaire d'autant qu'il contribuerait à améliorer le climat scolaire (Cohen, McCabe et al., 2009).

Dans ce contexte qui interroge "architecture ", " espaces scolaires» et "qualité de vie », nous avons réalisé une étude sur l'évaluation de la dimension architecturale des collèges français récents. C'est précisément auprès des principaux acteurs de ces lieux, principalement les élèves, que nous avons entrepris ce projet. Il s'agit alors d'obtenir 
leur ressenti à l'égard de leur bâtiment scolaire. Quel jugement portent-ils? Le trouvent-t-ils beau, agréable, moderne ou au contraire, ont-ils un avis plus négatif ? Enfin, comme le précisent Tcherkassof et Frijda (2014, p.529), "les ressentis émotionnels sont des perceptions de l'engagement dynamique du corps dans l'interaction ». Il y a l'idée que les émotions sont « des attitudes corporelles exprimant la relation du sujet à l'objet émotionnel» (Tcherkassof et Frijda, 2014, p. 529). Autrement dit, comme l'ont démontré Arnold (1960) ou Lazarus (1991), les émotions sont le fruit d'une influence mutuelle d'une personne et de son environnement (Lazarus et Launier, 1978). Ainsi, quel type d'émotions leur procure leur bâtiment scolaire? Il s'agit donc de questionner pour cet article l'environnement physique des collégiens.

\section{Méthodologie}

11 Pour répondre à ces questions, nous avons réalisé une enquête à la rentrée scolaire 2018, d'octobre à novembre, au sein de trois collèges publics aux caractéristiques semblables afin de limiter quelques variables parasites liées au contexte. Conscients que bon nombre de facteurs s'immiscent et impactent les réponses des sujets, nous avons priorisé une localisation péri-urbaine en province et des catégories socioprofessionnelles moyennes pour tous les établissements. 795 collégiens dont 383 filles et 412 garçons, ont participé à cette étude. Les répondants ont été répartis en deux catégories : tous les élèves de sixième et de troisième des trois établissements. Nous avons ainsi voulu analyser les différences éventuelles liées à deux principales variables explicatives : le genre et l'âge (en prenant les deux extrêmes, à savoir les plus jeunes et les plus âgés du collège). Au regard des perceptions distantes entre les filles et les garçons révélées par différentes enquêtes de victimation d'institutions nationales (Ministère de l'éducation nationale, Direction de l'évaluation, de la prospective et de la performance, 2011) et de chercheurs (Hébert, Dugas, 2017), il semble pertinent en effet d'interroger la variable " genre ». De même, il paraît opportun de convoquer la variable « âge » car " plus les acteurs sont jeunes, plus ils sont sensibles à leur environnement " (Pourchet, 2005, p. 3) et leur bien-être diminue avec l'ancienneté au collège (Hubert, 2013). Nous avons procédé à la passation des questionnaires à la population précitée en salle pupitre à partir du logiciel d'enquête statistique "lime survey » .

\section{Les caractéristiques architecturales des établissements évalués}

12 Notre choix s'est porté sur des établissements inaugurés il y a moins de cinq années. Sur le plan architectural, chacun renvoie au même modèle de construction. Il s'agit de collèges de type "cour » car c'est l'option largement répandue en France (Rigolon, 2010). Comme le précise Rigolon dans son rapport pour l'OCDE', cette architecture donne une place importante aux espaces extérieurs et utilise beaucoup de lieux de circulation. «Bien qu'il existe des variations, la principale caractéristique de ce type est une zone extérieure protégée facile à surveiller et psychologiquement rassurante " (Rigolon, 2010). En somme, nous nous situons sur un schéma classique d'établissements avec des couloirs alimentant des deux côtés des salles de classe et une vaste cour de récréation « bitumée » à l'extérieur des bâtiments. 
13 Malgré ces caractéristiques semblables, les trois collèges se distinguent sur certains aspects comme la place accordée à la lumière naturelle, au hall couvert ou aux espaces naturels (cf. tableau 1).

Tableau 1 - Comparaison des différentes caractéristiques environnementales des trois collèges évalués

\begin{tabular}{|l|l|l|l|}
\hline & Collège A & Collège B & Collège C \\
\hline Typologie & Cour & Cour & Cour \\
\hline Capacité d'accueil & 650 & 650 & 850 \\
\hline Taux d'occupation & $89 \%(580$ élèves) & $92 \%(600$ élèves) & $94 \%(800$ élèves) \\
\hline Façade & Bois & Bois & Brique \\
\hline Étage & 2 & 2 & 2 \\
\hline Parvis & Simple & Simple & Double \\
\hline Cour de récréation & Bitumée & Bitumée & Bitumée \\
\hline Lumière naturelle & Présente & Peu présente & Présente \\
\hline Hall & Oui & Oui & Non \\
\hline Sol naturel & Peu présent & Absent & Absent \\
\hline
\end{tabular}

\section{Les outils}

14 Comme le précise Ratiu (2003, pp. 85-86), «les démarches d'évaluation de l'environnement sont entreprises dans le but de réaliser l'adéquation entre les qualités environnementales et les attentes des publics, leur préservation ainsi que la construction des standards et normes les concernant ». Or, d'ici une dizaine d'années, près de quatre mille collèges devront être construits ou rénovés en France. C'est pourquoi, l'objectif est "d'obtenir des informations valides, fidèles et pertinentes » (Ratiu, ibid.) afin que les architectes et ceux qui orientent les politiques de construction participent à l'amélioration de la qualité de vie des élèves.

Notre méthode d'évaluation s'est donc voulue centrée sur l'analyse des qualités environnementales des collèges récemment construits à partir d'une démarche par questionnement indirect afin de limiter les biais classiques liés aux questions directes (Grémy, 1992), dont les répondants maîtrisent le résultat final.

Pour construire notre outil, nous nous sommes appuyés largement sur les travaux de Hesselgren (1987, cité par Uzell et Romice, 2003). Il «a créé une liste relativement exhaustive d'adjectifs bipolaires pour décrire les réactions des gens envers un objet, en distinguant les réactions descriptives, émotionnelles et évaluatives » (Uzzel et Romice, 2003, p. 78). Pour ses travaux, le chercheur s'était largement appuyé sur les adjectifs 
sémantiques établis par Küller (1980) et sur les émotions primaires identifiées par Plutchik (1974).

17 Conservant la même structure, nous avons, quant à nous, opté pour les échelles du différentiateur sémantique d'Osgood (1957) car la pertinence et la validité du choix des échelles retenues ont déjà été testées pour d'autres travaux (Fischer, 1989; Collard, 1998; Dugas, 2009, etc.). Cette technique de contournement (procédure de questionnement indirect) permet «au chercheur d'explorer de façon indirecte les significations subjectives que chaque individu associe aux stimuli de son environnement social» (Parlebas, 1994, p.9). Autrement dit, on interprète la perception subjective des répondants, ici sur leur bâtiment scolaire, en leur demandant de réagir avec rapidité, comme on le ressent, en cochant des cases liées à des échelles composées à leurs extrémités de deux adjectifs antonymes. Le stimulus inducteur choisi contient une signification connotative que l'on regroupe dans trois dimensions - La valeur (V), La puissance (P) et l'activité (A), en réarrangeant les échelles choisies pour l'enquête. Dans notre enquête, on recherche les réponses spontanées, affectives (et non pas rationnels) des collégiens associés à leur bâtiment scolaire.

Ces trois dimensions résultent d'une enquête réalisée par Osgood (1957) dans laquelle il demandait aux répondants de se positionner à l'aide d'une cinquantaine d'échelles, composées d'adjectifs antonymes, sur une vingtaine de mots (stimuli inducteurs). L'analyse factorielle du traitement des données, réalisée par Osgood, révèle que les échelles peuvent être regroupées en trois dimensions distinctes. La principale - celle qui possède le plus de poids (33,8 \% de l'information totale) - est la « valeur » (V). Elle renvoie à la manière dont un individu perçoit les choses. C'est ce que les sociologues nomment l'image. Elle correspond à des connotations de "plaisir/déplaisir". Nous avons choisi - parmi les paires d'adjectifs réunies par Osgood dans cette dimension -, les adjectifs laid/beau, désagréable/agréable, sale/propre, raté/réussi et déplaisant/ plaisant. La deuxième dimension est la "puissance» $(\mathrm{P})$. C'est l'effort imposé par l'adaptation à la situation suggérée qui définit cette dimension. Elle est principalement associée à des caractéristiques de force, de masse et d'énergie (Parlebas, 1994). Pour anecdote, un associé d'Osgood l'a surnommée «le facteur du joueur de foot ». Pour notre recherche, les paires silencieux/bruyant, pâle/coloré, fragile/résistant et sombre/éclairé nous sont apparues pertinentes à choisir. Enfin, la troisième dimension est l'activité (A). Elle correspond à la rapidité de l'ajustement, «il dépend de la perception des traits de mobilité du stimulus inducteur» (Ibid., p. 14). Nous avons choisi parmi la liste des possibles les adjectifs calme/excitable, froid/chaud, fermé/ ouvert et dangereux/sûr.

Pour résumer, le facteur "Valeur » possède le plus de poids (33,8 \% de l'information totale) contrairement aux deux autres facteurs, "Puissance » et "Activité » qui représentent seulement 13,2\% de l'information totale (respectivement $7 \%$ et 6,2\%). Ainsi, la dimension « valeur » totalise, à elle seule, $70 \%$ de la variance totale et les trois facteurs prennent en charge $50 \%$ de la variance totale. Ce qui expliquera plus loin dans les résultats, l'attention et l'analyse plus poussées et portées à ce facteur. Les deux derniers facteurs seront néanmoins fort utiles pour pondérer, compléter et affiner nos interprétations issues des résultats de la dimension "Valeur». Elles reflèteront principalement les connotations liées au type d'architecture choisi pour construire des espaces de vie scolaire. 
20 Nous avons ainsi choisi treize échelles (réparties dans trois dimensions) parmi la cinquantaine testée et validée par Osgood (1957). Cette procédure peut offrir des données originales et complémentaires dès lors que l'on s'intéresse aux représentations des enquêtés.

21 Signalons en dernier lieu que nous avons pris soin de préciser aux répondants que l'on voulait étudier la signification, le sens qu'ils donnaient au stimulus «bâtiment scolaire ", et leur ressenti associé, il se peut que des effets de contexte (climat scolaire dégradé, élèves en décrochage, équipes enseignante changeantes, etc.) aient pu impacter les réponses des collégiens. C'est pourquoi, fort de ces premiers résultats, nous projetons, dans de futures enquêtes, de confronter ces tendances qui émergent en augmentant la volumétrie des répondants, en comparant les variables démographiques (milieux rural, urbain et péri-urbain), socioculturelles (REP+, favorisé) et architecturales.

Le recueil des données est réalisé en associant (après coup) à chacune des sept cases de chaque échelle, une valeur s'étalant de -3 (pôle le plus négatif) à +3 (pôle le plus positif), respectant la distribution de la procédure d'Osgood issue de l'analyse factorielle. Dès lors, nous pouvons calculer l'écart-type et la moyenne des connotations par dimension et par échelle. Concernant les questions consacrées aux émotions éveillées par le bâtiment, nous avons opté pour une échelle de Likert. De la même manière, nous avons procédé aux calculs des moyennes et des écarts-type.

$23 \mathrm{Au}$ regard de ces cadrages théoriques et méthodologiques, découlent plusieurs hypothèses. En premier lieu, à l'instar de Chiva et Dubost (1990, p. 10), tout jugement de goût repose sur l'intériorisation de normes et de valeurs et n'échappent aux " normes du goût dominant et aux modes du moment ». Au regard des échelles constitutives de la dimension "valeur » du différenciateur d'Osgood (beau / laid ; agréable / désagréable, etc.) tout laisse à penser que les élèves jugeront positivement leur récent collège. En second lieu, et au vu de l'état de l'art donné à lire en amont, nous soumettons l'hypothèse selon laquelle l'âge et le genre des élèves engendrent des évaluations de l'architecture scolaire distinctes. Enfin, des différences d'évaluation entre les trois établissements peuvent se cristalliser autour de facteurs d'influence négative (bruit, lumière, etc.)

\section{Résultats et discussion}

\section{Résultats globaux}

En premier lieu, les résultats révèlent d'un point de vue général des évaluations positives concernant l'environnement scolaire des élèves (cf. tableau 2 ci-après). Les collèges sont en effet plutôt appréciés sur le plan bâtimentaire $(3,45$ / 5 sur une échelle de Likert $^{8}$ ) et plusieurs espaces harmonieux à l'intérieur de chacun d'entre eux sont admis par les répondants $(3,18 / 5)$. Ces espaces sont même davantage valorisés que les lieux disharmonieux. Néanmoins, la stigmatisation de quelques espaces nous rappelle que certains endroits, notamment la cour de récréation et les espaces interstitiels sont souvent laissés pour compte par les architectes (Hornqvist, 2001) et par les politiques éducatives alors qu'ils sont ressentis par les élèves comme anxiogènes (Hébert, Dugas, 2017). 
Tableau 2 - Ressenti de tous les collégiens sur leur bâtiment scolaire. L'échelle oscille de 0 à 5 $(0:$ : pas du tout » et $5:$ « énormément »).

\begin{tabular}{|l|l|l|l|}
\hline $\begin{array}{l}\text { Le ressenti des collégiens des 3 } \\
\text { établissements }(\boldsymbol{n}=\mathbf{7 9 5})\end{array}$ & $\begin{array}{l}\text { Apprécies-tu ton } \\
\text { collège ? }\end{array}$ & $\begin{array}{l}\text { Présence d'espaces } \\
\text { harmonieux }\end{array}$ & $\begin{array}{l}\text { Présence d'espaces } \\
\text { non harmonieux }\end{array}$ \\
\hline Moyenne & 3,45 & 3,18 & 2,36 \\
\hline Ecart-type & 0,92 & 1,10 & 1,15 \\
\hline
\end{tabular}

Cette première tendance est confortée par les données récoltées à partir des échelles sémantiques puisqu'elles font état d'une connotation générale proche de $+1(+0,92)$. Plus précisément, l'image (Valeur) des collèges est positive $(+1,24)$ comme en atteste en détail la figure 1 ci-dessous. Les écarts-type $(1,23)$ restent néanmoins élevés ce qui signifie des disparités importantes chez la totalité des collégiens interrogés $(\mathrm{n}=795)$.

Figure 1 - Différenciateur d'Osgood : la dimension "Valeur " pour le stimulus « Ce que tu vois à propos de ton collège »

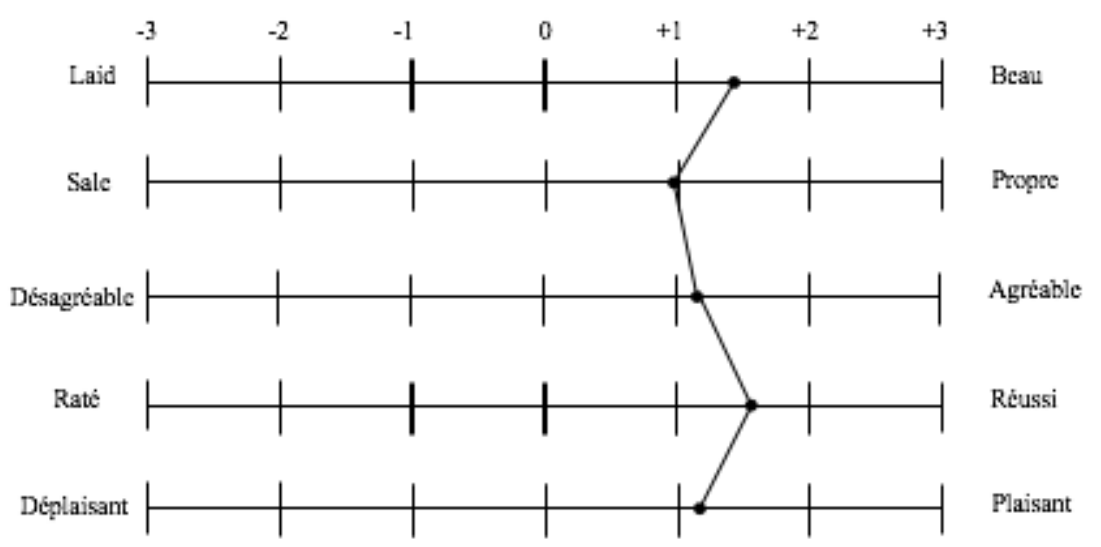

En second lieu, le collège éveille davantage d'émotions positives que d'émotions négatives. Ainsi, nous observons que le bâtiment suscite plus de joie (2,98 sur 5$)$, d'approbation ( 3,01 sur 5$)$, d'attente (2,58 sur 5 ) que de colère ( 1,75 sur 5$)$, de peur $(1,54$ sur 5$)$, de tristesse (1,59 sur 5$)$ ou de rejet (1,68 sur 5). Observons alors s'il existe des corrélations entre les connotations exprimées par les collégiens à travers les échelles sémantiques et les émotions suscitées par l'environnement scolaire (cf. tableau 3). Ainsi, nous partons du postulat que les émotions sont un facteur explicatif du comportement humain et que l'évaluation que l'organisme fait d'un stimulus, d'un évènement ou d'une situation détermine le déclenchement d'une émotion.

Tableau 3 - Corrélations entre les échelles du différenciateur sémantique et les émotions suscitées par l'établissement.

\begin{tabular}{|l|l|l|l|}
\hline & Valeur & Puissance & Activité \\
\hline
\end{tabular}




\begin{tabular}{|l|l|l|l|}
\hline Colère & $-0,39^{*}$ & $-0,19^{*}$ & $-0,27^{*}$ \\
\hline Joie & $0,44^{*}$ & $0,21^{*}$ & $0,31^{*}$ \\
\hline Peur & $-0,15^{*}$ & $-0,06^{*}$ & $-0,09^{*}$ \\
\hline Approbation & $0,22^{*}$ & $0,07^{*}$ & $0,15^{*}$ \\
\hline Surprise & $0,25^{*}$ & $0,15^{*}$ & $0,23^{*}$ \\
\hline Tristesse & $-0,26^{*}$ & $-0,08^{*}$ & $-0,12^{*}$ \\
\hline Rejet & $-0,36^{*}$ & $-0,20^{*}$ & $-0,23^{*}$ \\
\hline Attente & $0,38^{*}$ & $0,26^{*}$ & $0,29^{*}$ \\
\hline
\end{tabular}

(* : signification statistique : $p<0,05)$

Le calcul des coefficients de corrélations de Pearson atteste d'une relation significative entre l'évaluation de l'établissement et les émotions suscitées par cet endroit. Nous observons néanmoins que les dimensions «Puissance " et «Activité » sont corrélées moins fortement avec les émotions que la dimension «Valeur». Nous constatons donc un lien plus fort entre l'image de l'établissement et les émotions procurées par ce bâtiment. Par ailleurs, l'image (Valeur) de l'établissement corrèle positivement avec des émotions positives (joie, approbation, surprise, attente) et corrèle négativement avec les émotions négatives (colère, peur, tristesse, rejet). Autrement dit, comme vu en introduction, nos résultats attestent du lien entre les émotions d'un individu et son environnement (Arnold, 1960, Lazarus, 1991). Plus largement, certaines conditions environnementales seraient potentiellement propices à provoquer des émotions négatives. Pour rappel, nous savons par exemple que la densité, le bruit ou la chaleur sont propices au stress (Moser, 2009). En somme, comme le précise Nordström (2010), «l'environnement acquiert pour les enfants une valeur émotionnelle parce qu'ils sont capables de développer et d'approfondir des manières d'y être sensible ».

Ces premiers résultats montrent des connotations plutôt positives à l'égard de l'architecture des collèges même si les élèves ont aussi identifié des espaces non harmonieux (cf. tableau $1 ; 2,36 / 5$ ). Pour autant, les écarts type élevés attestent d'une certaine hétérogénéité chez nos répondants. Par conséquent, regardons si ces différences se cristallisent autour des variables genre et âge.

\section{Des différences en fonction des variables genre et âge ?}

29 À la lecture du tableau 4, nous observons des différences significatives entre les garçons et les filles concernant la valeur accordée au bâtiment. Ainsi, les filles s'illustrent par un ressenti plus positif à l'égard des collèges $(+1,36$ contre $+1,11)$. Les écarts-type révèlent même davantage d'homogénéité chez elles. Quant aux émotions suscitées par l'établissement scolaire, seuls les résultats concernant la colère et le rejet sont significativement différents (à $\mathrm{p}<0,5)$; les garçons exprimant davantage ces émotions négatives ( 1,9 contre 1,6 pour la colère $; 1,81$ contre 1,56 pour le rejet). 
Tableau 4 - Connotations exprimées par les garçons et les filles à l'égard de leur collège

\begin{tabular}{|c|c|c|c|c|}
\hline & $\begin{array}{l}\text { Apprécies-tu ton } \\
\text { collège? }\end{array}$ & $\begin{array}{l}\text { Dimension } \\
\text { Valeur }\end{array}$ & $\begin{array}{l}\text { Dimension } \\
\text { Puissance }\end{array}$ & $\begin{array}{l}\text { Dimension } \\
\text { Activité }\end{array}$ \\
\hline $\begin{array}{l}\text { Garçons } \\
(n=383)\end{array}$ & & & & \\
\hline Moyenne & 3,58 & $+1,11$ & $+0,88$ & $+0,63$ \\
\hline Ecart-type & 1 & 1,29 & 0,92 & 0,95 \\
\hline Filles $(n=412)$ & & & & \\
\hline Moyenne & 3,51 & $+1,36$ & $+0,95$ & $+0,59$ \\
\hline Ecart-type & 0,85 & 1,17 & 0,89 & 0,87 \\
\hline Test T (p) B/C & 0,16 & $0,00 *$ & 0,40 & 0,69 \\
\hline
\end{tabular}

$(*$ : signification statistique : $p<0,05)$

30 Si nos résultats montrent des différences significatives entre les garçons et les filles autour de l'image de l'établissement, le tableau 5 ci-dessous révèle des différences significatives entre les plus jeunes collégiens et les plus âgés au sujet des trois dimensions du différenciateur, comme aussi sur l'appréciation du collège. Ainsi, les élèves de $6^{\text {è }}$ connotent plus positivement le bâtiment que les $3^{\text {èmes }}$. Autrement dit, ces jugements contrastés liés à l'âge pourraient trouver une explication dans la citation suivante de Nordström :

$\mathrm{Au}$ fur et à mesure que les enfants avancent en âge, et que leurs capacités mentales s'accroissent, leurs relations avec l'environnement physique changent et deviennent plus conceptuelles. Un net changement dans leurs relations avec l'environnement se produit à la puberté, non seulement sous l'influence du développement mental, mais aussi du développement social et affectif, et de leurs expériences de l'environnement (Nordström, 2010).

Tableau 5 - Connotations exprimées par les élèves en fonction de leur âge à l'égard de leur collège

\begin{tabular}{|l|l|l|l|l|}
\hline & $\begin{array}{l}\text { Apprécies-tu ton } \\
\text { collège? }\end{array}$ & $\begin{array}{l}\text { Dimension } \\
\text { Valeur }\end{array}$ & $\begin{array}{l}\text { Dimension } \\
\text { Puissance }\end{array}$ & $\begin{array}{l}\text { Dimension } \\
\text { Activité }\end{array}$ \\
\hline $\begin{array}{l}\text { Élèves de } \\
(\mathbf{n}=\mathbf{3 8 2})\end{array}$ & & & & \\
\hline Moyenne & 3,81 & $+1,64$ & $+1,15$ & $+0,95$ \\
\hline Ecart-type & 0,88 & 1,16 & 0,81 & 0,88 \\
\hline & & & & \\
\hline
\end{tabular}




\begin{tabular}{|l|l|l|l|l|}
\hline $\begin{array}{l}\text { Élèves de 3è } \\
(\mathbf{n}=\mathbf{4 1 3})\end{array}$ & & & & \\
\hline Moyenne & 3,12 & $+0,87$ & $+0,73$ & $+0,28$ \\
\hline Ecart-type & 0,84 & 1,19 & 0,93 & 0,80 \\
\hline Test T (p) B/C & $0,00^{*}$ & & & $0,00^{*}$ \\
\hline
\end{tabular}

(* : signification statistique : $p<0,05)$

31 Par ailleurs, comme le soumet Escofet (2011), le collège regroupe des élèves dont les valeurs juvéniles sont en contradiction avec celles défendues par l'institution scolaire. Alors que les espaces (classe 稂 couloirs) viennent symboliser cette opposition (Monnard, 2015), nous soumettons l'hypothèse selon laquelle la critique du bâtiment vient surligner cette distance envers les valeurs scolaires.

Enfin, lorsque nous croisons les variables "âge » et " genre " (cf. figure 2 ci-dessous), nous constatons que les plus jeunes filles ont une image significativement plus positive du collège que les autres élèves (à $p\left[{ }_{[}[0,05)\right.$. À l'inverse, l'histogramme atteste que les garçons les plus âgés sont les plus critiques à l'égard de leur bâtiment scolaire (à $p$ 㹂0,05).

Figure 2 - Résultats du différenciateur sémantique en fonction de l'âge et du genre

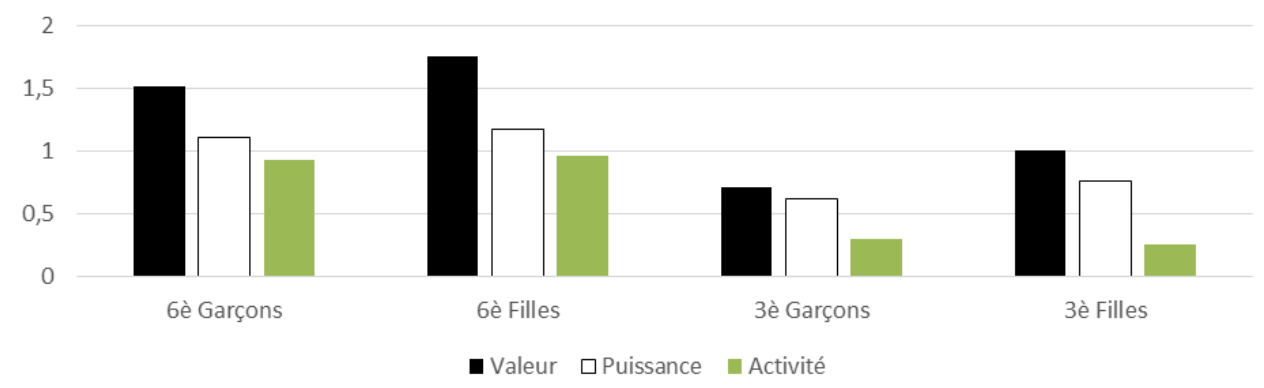

Nous avons pris soin de souligner en méthodologie que si les collèges choisis pour cette étude avaient pour point commun la typologie architecturale, pour autant, quelques éléments divergeaient. Considérant qu'un certain nombre de facteurs architecturaux (bruit, lumière, etc.) peuvent influencer négativement les comportements des individus (Moser, 2009, Fischer, 2011), il s'agit désormais d'observer si d'un établissement à l'autre, ces facteurs sont mis en évidence.

\section{Des différences entre établissements ?}

En premier lieu, le tableau 6 ci-dessous révèle des connotations positives pour les trois collèges. Néanmoins, le test de Student réalisé deux à deux fait état de différences significatives de ressenti entre les collèges $A$ et $B$ puis $A$ et $C$ sur l'ensemble des dimensions. Quant aux collèges B et C seules les dimensions « Puissance » et " Activité » 
affichent des distinctions significatives. D'un point de vue général, le collège A est largement connoté positivement par rapport aux deux autres établissements.

Tableau 6 - Connotations exprimées par les élèves au regard des trois collèges étudiés

\begin{tabular}{|c|c|c|c|c|}
\hline & $\begin{array}{l}\text { Apprécies-tu ton } \\
\text { collège? }\end{array}$ & $\begin{array}{l}\text { Dimension } \\
\text { Valeur }\end{array}$ & $\begin{array}{l}\text { Dimension } \\
\text { Puissance }\end{array}$ & $\begin{array}{l}\text { Dimension } \\
\text { Activité }\end{array}$ \\
\hline $\begin{array}{l}\text { Collège } \\
(n=215)\end{array}$ & & & & \\
\hline Moyenne & 3,77 & $+1,86$ & $+1,22$ & $+0,89$ \\
\hline Ecart-type & 0,83 & 0,13 & 0,96 & 0,81 \\
\hline $\begin{array}{l}\text { Collège } \\
(n=218)\end{array}$ & & & & \\
\hline Moyenne & 3,33 & $+0,97$ & $+0,61$ & $+0,34$ \\
\hline Ecart-type & 1 & 0,14 & 0,71 & 0,71 \\
\hline $\begin{array}{l}\text { Collège } \\
(n=362)\end{array}$ & & & & \\
\hline Moyenne & 3,33 & $+1,04$ & $+0,91$ & $+0,58$ \\
\hline Ecart-type & 0,91 & 0,4 & 1,26 & 0,36 \\
\hline Test T (p) A/B & $0,000^{*}$ & $0,000^{*}$ & $0,000^{*}$ & $0,000^{*}$ \\
\hline Test T (p) A/C & $0,000^{*}$ & $0,000^{*}$ & $0,000^{*}$ & $0,000^{*}$ \\
\hline Test T (p) B/C & 0,988 & 0,482 & $0,000^{*}$ & $0,002^{*}$ \\
\hline
\end{tabular}

(* : signification statistique : $p<0,05)$

En second lieu, les différentes échelles sémantiques (figure 3 ci-dessous) permettent d'une part, de conforter les différences suggérées précédemment entre les trois collèges et, d'autre part, mettent en lumière la présence de perturbateurs environnementaux, nuisibles au bien-être des élèves. 
Figure 3 - Les échelles sémantiques sur le stimulus "Ton collège, ce que tu vois » entre les élèves de trois collèges différents (Collège $A, B$ et $C$ )

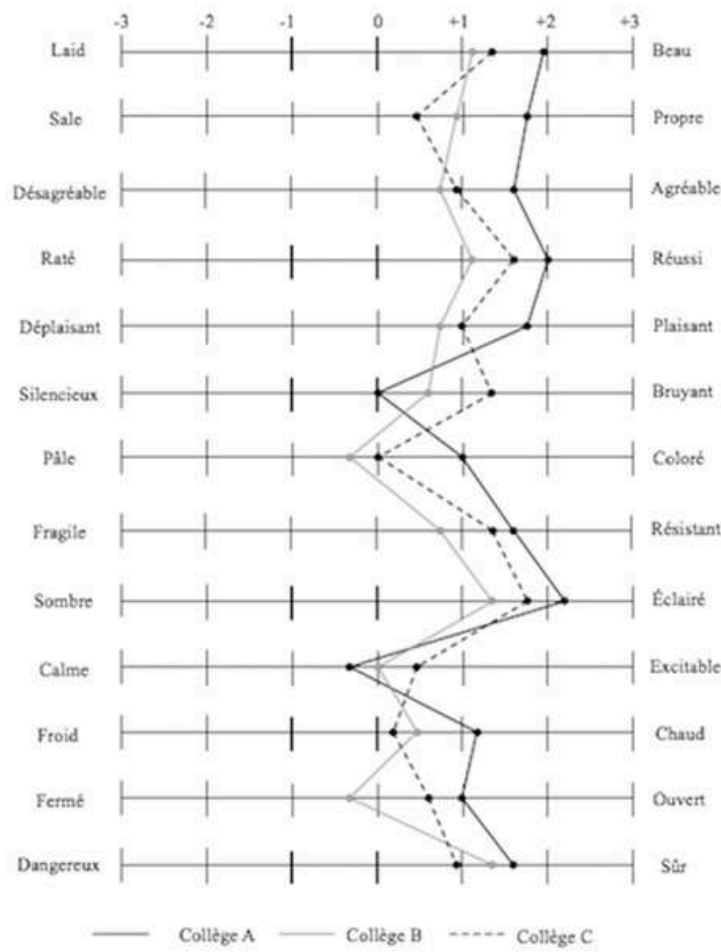

Le premier facteur négatif est le bruit. Nous constatons que seul le collège A affiche une connotation neutre, proche de zéro $(+0,03)$, sur l'échelle «silencieux / bruyant». Autrement dit, les élèves des collèges $B$ et $C$ se distinguent significativement en stigmatisant les nuisances sonores dans leur établissement. Ce constat interroge forcément puisque nous savons par exemple que les élèves qui se trouvent dans des écoles bruyantes s'avèrent plus facilement distraits par des bruits de fond, abandonnent plus rapidement que les autres élèves les tâches complexes (Cohen et al., 1980), éprouvent des difficultés d'apprentissage de la lecture et échouent significativement plus souvent à des problèmes d'arithmétique (Moch, 1981). «En outre, le bruit semble avoir des effets durables car on constate que les enfants ne s'y adapte pas. Ils continuent à en être affectés, même s'ils n'en parlent plus » (Fischer, 2011, p. 122).

Le deuxième facteur d'influence négatif identifié à partir de la figure 3 est l'aménagement intérieur symbolisé par les couleurs et la lumière. Concernant la couleur, le collège A se distingue significativement de ces deux homologues par une valorisation des couleurs alors que pour la lumière, c'est plutôt le collège B qui s'avère plus sombre. Il est vrai que nous avions pu noter que cet établissement se distinguait par la faible place donnée à la lumière naturelle, notamment dans les espaces de transition. Ce constat n'est pas sans effet puisqu'un lien a été établi entre l'éclairage artificiel et les symptômes de dépression (Riemersma-van der Lek, 2008).

Enfin, nous aimerions porter un regard sur la paire d'adjectifs "fermé / ouvert ». Le collège, comme tout espace institutionnel partiel (Goffman, 1973), renvoie l'image d'un espace séparé marqué " par une division fondamentale entre un dehors et un dedans " (Fischer, 2011, p. 158). En instaurant des parvis ou double parvis (comme le collège C), 
se trouve à peine dissimulée la volonté chez les décideurs de renforcer les frontières physiques et psychologiques entre le dehors et le dedans afin de de marquer un lieu sécurisant, en d'autres termes un sanctuaire. Nos résultats semblent abonder dans ce sens puisque les élèves interrogés sont assez contrastés sur les adjectifs «fermé / ouvert ». C'est plus particulièrement le collège B qui se distingue significativement des deux autres établissements car ce dernier est ressenti comme fermé. Ainsi, comme le précise Depoilly (2015), les filles, comme les garçons peuvent vivre le collège comme un espace d'emprisonnement et d'infantilisation. Il s'agit dès lors que l'architecture du collège ne participe pas de ce phénomène. Or, de récents travaux (Hébert, à paraître, 2018a) révèlent que la surveillance et le contrôle des élèves sont largement intégrés dans les constructions d'établissements scolaires. Si le bâtiment est réfléchi comme un lieu propice aux apprentissages et au bien-être des élèves, les effets sur les élèves de la structure panoptique et des aménagements dédiés à la surveillance sont de l'ordre du pouvoir physique et moral sur eux (de Champs, 2010) et du sentiment de captivité. En somme, la question du malentendu entre des adultes soucieux d'instaurer des espaces " régis par des critères d'organisation et de sécurité " (Levrard et Delalande, 2015, p. 133) et des enfants en attente d'espace de liberté, d'échanges mérite d'être prise en considération dans les projets de construction d'établissements scolaires.

\section{Conclusion}

Nous avons cherché à travers cette enquête à poser les bases d'une réflexion entre l'espace conçu et l'espace vécu. Autrement dit, il s'agissait pour des collégiens d'exprimer leur ressenti sur leur bâtiment scolaire. Trois tendances ont pu être identifiées. En premier lieu, de manière générale, les élèves connotent positivement leur établissement et ce malgré des différences entre garçons et filles et entre $6^{\grave{e}}$ et $3^{\text {è }}$. En second lieu, la perception des élèves à l'égard de leur bâtiment scolaire est fortement corrélée aux émotions éveillées par ce lieu. En quelque sorte, «une évaluation significative de l'environnement active une réaction émotionnelle appropriée au contexte » (Tcherkassof, 2008, p. 39). Enfin, les différences constatées entre les trois collèges analysés ont fait émergé pour certains d'entre eux, d'une part, des facteurs architecturaux d'influence négative du bien-être comme le bruit, l'absence de couleur et de lumière naturelle et, d'autre part, la problématique de l'« école sanctuaire » ou des « espaces de liberté ».

Cette étude permet ainsi de savoir comment les élèves, voient, perçoivent et évaluent le lieu dans lequel ils sont censés apprendre et être socialisés. Il s'agit ainsi d'apporter modestement, avec les précautions d'usage d'une enquête de terrain quelques connaissances sur l'environnement bâti afin d'impulser un processus de conception d'établissements scolaires où les élèves sont enfin pris en considération. C'est notamment par cette phase que pourra s'opérer la transformation de l'architecture scolaire. La tâche ne sera pas aisée. Car, comme le soumet Weiss (2003, p. 359) à propos du développement durable, la qualité de vie à l'école «se heurte à un dilemme... Quel comportement adopter, quelle décision prendre lorsque les intérêts individuels et collectifs sont contradictoires? ». En d'autres termes, comment concilier des bâtiments sécurisés tout en garantissant et valorisant la liberté des élèves ? Comment réduire les coûts de construction dont les financeurs sont si soucieux et privilégier des matériaux isolants et des aménagements spatiaux permettant aux enseignants de mettre une 
pédagogie en phase avec les enjeux du 21è siècle? Aussi, cette étude a le mérite d'interroger une co-construction bottom-up, associant les usagers dans toute démarche procédurale. Le bonheur des citoyens passe par leur participation active à la vie et décisions des politiques de la cité et des territoires, dont celle-ci, consacrée à l'éducation.

41 Si l'originalité de cette recherche réside dans le fait que les bâtiments scolaires n'aient jamais été évalués de la sorte, le bien-être à l'école ne peut pour autant être réduit simplement à l'impact de l'environnement sur les individus. D'autres facteurs en interaction sont en effet à considérer pour construire une école du bonheur, l'approche systémique, voire écosystémique semble indispensable pour tout approfondissement ultérieur. Le développement de l'estime de soi, de l'empathie, de la créativité ou la valorisation de la coopération entre les élèves constituent aussi de véritables enjeux pour nos futurs élèves. Le bâtiment scolaire se doit alors d'être au service de cette mise en jeu pédagogique comme l'a déjà entrepris un certain nombre de pays telle que la Finlande.

\section{BIBLIOGRAPHIE}

Arnold, M.B. (1960). Emotion and personality. New York: Columbia.

Barett, P., Davies, F., Zhang, Y., Barett, L. (2015). The impact of classroom design on pupils' learning : Final results of a holistic, multi-level analysis. Building and Environment, 89, 118-133.

Barker, R.G. \& Gump, P.V. (1964). Big school, small school : High school size and Student behavior. Stanford : Stanford University Press.

Bronzaft, A.L. \& McCarty, D.P. (1975). The effect of elevated train noise on reading ability. Environment and Behavior, vol. (7), 4, 517-527.

Châtelet, A.-M. (2004). L'architecture des écoles au XXe siècle. Histoire de l'éducation, vol. (102) En ligne : https://journals.openedition.org/histoire-education/696

Cohen, J., McCabe, E. M., Michelli, N. M., \& Pickeral, T. (2009). School climate : Research, policy, teacher education and practice. Teachers College Record, vol. (111), 1, 180-213. En ligne : http:// www.tcrecord.org/Content.asp?ContentId $=15220$

Collard, L. (1998). Sports, enjeux et accidents. Paris : Puf.

Debarbieux, E. (2011). Enquête nationale de victimation et climat scolaire auprès d'enfants âgés de 8 à 12 ans. A l'école des enfants heureux... enfin presque. En ligne : http://www.unicef.fr/ userfiles/UNICEF_FRANCE_violences_scolaires_mars_2011.pdf Depoilly, S. (2015). Filles, garçons... et les interstices de l'école. Diversité, 179, 105-109.

Derouet-Besson, M.-C. (2007). Vivre ensemble dans les bâtiments scolaires : un défi permanent. Diversité, 150, 127-132.

Divay, al. (2004). Projet pilote de revitalisation intégrée. Démarche d'évaluation. Montréal : INRS Urbanisation, Culture et Société. 
Dizerbo, A. (2017). Quels murs pour quelle école ? Architecture scolaire, normes scolaires et figures d'élèves. Revue Éducation et socialisation - Les cahiers du Cerfee, 43, En ligne, https:// journals.openedition.org/edso/1915

Dugas, E. (2009). L'influence d'un stage de formation Continue en EPS : un autre regard sur les jeux traditionnels. Revue Savoirs, 20, 134-158.

Escofet, P. (2011). Le microcosme juvénile. Sociabilité adolescente, école et violences. Gollion : Infolio.

Fischer, G.N. (1989). Psychologie des espaces de travail. Paris : Armand Colin.

Fischer, G.N. (2011). Psychologie sociale de l'environnement. Paris : Dunod.

Forster, S. (2004). Architecture scolaire : regard historique tourné vers l'avenir. Bulletin CIIP, 15, En ligne : https://www.irdp.ch/data/secure/440/document/architecture-scolaire-440.pdf

Guimard, P. \& al. (2015). Le bien-être des élèves à l'école et au collège. Validation d'une échelle multidimensionnelle, analyses descriptives et différentielles. In Moisan, C., et al. Climat scolaire et bien-être à l'école, Educations \& Formations, 163-185 Grangaard, E.M., (1995), « Color and Light Effects on Learning ", Présenté à Association for Childhood Education International Study Conference and Exhibition (Washington, DC, April 12-15, 1995).

Grémy J.P. (1992). La formulation des questions d'enquête : son effet sur les réponses. In La qualité de l'information dans les enquêtes. Paris : Dunod, 97-114.

Hébert, T. (2018). Comment ça va à l'école ? Les cahiers pédagogiques, 48, Hors-série numérique Espaces et architectures scolaires, 31-33.

Hébert, T. (2019). La sécurité dans les établissements de l'enseignement secondaire sous le prisme de l'architecture et l'aménagement des espaces. La recherche en éducation.

Hébert, T. \& Dugas, É. (2017). Quels espaces scolaires pour le bien-être relationnel ? Enquête sur le ressenti des collégiens français. Revue Éducation et socialisation - Les cahiers du Cerfee, 43, En ligne, https://journals.openedition.org/edso/1904.

Hesselgren, S. (1987). On architecture. An architecture theory based on psychological research. Lund : S.H. And Student Literature.

Hubert, T. (2013). La Victimation des collégiens en milieu scolaire. (Rapport pour l'enquête de victimation organisée par l'Education nationale).

Jackson, T. (2002). Quality of life, Economic Growth and Sustainability. In M. Cahill et A. Fitzpatrick (eds.) Environment and Welfare : towards a green social policy (97-116). London : Palgrave Macmillan.

Layard, R. (2007). Le prix du bonheur : Leçons d'une science nouvelle. Paris : Armand Colin.

Lazarus, R.S. (1991). Emotion and adaptation. New York : Oxford University Press.

Lazarus, R.S. é Launier, R. (1978). Stressrelated transactions between persons and environment. In L. Perwin et M. Lewis, Perspective in interactional psychology (287-327) New York : Plenum Press.

Levrard, S. \& Delalande, J. (2015). Mon école, c'est pas que je ne la trouve pas belle, c'est qu'on est ensemble. Diversité, 179, 131-135.

Lévy-Leboyer (1980). Psychologie et environnement. Paris : PUF.

Loudier, C. \& Vallet B. (2010). L'influence de la sécurité sur la conception urbaine. Les Cahiers de l'Institut d'Aménagement et d'Urbanisme de la région Ile-de- France, 155, 25-28.

Mazalto, M. (2013). Les cours de récréation. Paris : Fabert. 
Moch, A. (1985). La sourde oreille : grandir dans le bruit. Toulouse : Privat.

Monnard, M. (2015). Entre deux salles de classe, parcourir l'école. Diversité, 179, 98-103.

Moscovici, S. (1988). Psychologie sociale. Paris : PUF.

Moser, G. (2009). Psychologie environnementale. Les relations homme-environnement. Bruxelles : De Boeck.

Newman, O. (1972). Defensible Space. People and Design in the Violent City. London: The architectural Press.

Nordström, M. (2010). L'utilité des cours dans la construction de l'identité de genre : observations des activités pratiquées par les filles et les garçons âgés de 12 ans pendant la récréation. In I. Danic et al. (dir.) Enfants et jeunes dans les espaces du quotidien (21-34). Rennes : PUR.

Osgood, C., Suci, G. \& Tannenbaum, P. (1957). The measure- ment of meaning. Illinois : University of Illinois Press.

Paquot, T. (2015). Désastres urbains. Les villes meurent aussi. Paris : La découverte.

Parlebas, P. (1994). Le différenciateur sémantique. Applications sociologiques, In Publications de $3 e$ cycle de l'UFR de Sciences sociales (7-29), Université Paris V.

Plutchik, R. (1974). Foundations of experimental research. New York : Harper and Row.

Pourchet, G. (2005). Construire pour enseigner. Revue de l'Inspection générale, vol. (2), 3.

Ratiu, E. (2003). L'évaluation de l'environnement. In G. Moser et K. Weiss, Espaces de Vie. Aspects de la relation homme-environnement (85-112). Paris : Armand Colin.

Raibaud, Y. (2012). Genre et espaces du temps libre. L'information géographie, 2, vol. (76), 40-56.

Riemersma-van der Lek, R.F. \& al. (2008). Effect of Bright Light and Melatonin on Cognitive and Noncognitive Function in Ederly Residents of Group Care Facilities. A Randomized Controlled Trial. American Medical Association, JAMA, vol. (299), 22, 2642-2655.

Rigolon, A. (2010). Les plans de construction des écoles européennes du XXIe siècle :

présentation », OCDE, En ligne : http://www.oecd.org/fr/education/innovation-education/ centrepourdesenvironnementspedagogiquesefficacescele/44708525.pdf

Stockdale, J.E. (1978). Crowding : Determinants and Effects. Advances in Experimental Social Psychology, II, New York : Academic Press, 197-247.

Tcherkassof, A. (2008). Les émotions et leurs expressions. Grenoble : PUG.

Tcherkassof, A \& Frijda, N.H. (2014). Les émotions : une conception émotionnelle. L'année psychologique, 3, vol. (114), 501-535.

Toulier, B. (1982). L'architecture scolaire au XIXe siècle : de l'usage des modèles pour l'édification des écoles primaires. Histoire de l'éducation, 17, 1-29.

Uzzell, D. \& Romice, O. (2003). L'analyse des expériences environnementales. In G. Moser et K. Weiss, Espaces de Vie. Aspects de la relation homme-environnement (49-83). Paris : Armand Colin.

\section{NOTES}

1. Revue Éducation et socialisation (2017) coordonnée par... et plus récemment le numéro thématique Hors-série des cahiers pédagogiques consacré à ... (2018), Rapport du cnesco... 
2. Plusieurs colloques sur le bien-être à l'école ont récemment proposé des sympodiums sur l'architecture et les espaces scolaires (Nantes, 2017, etc.)

3. Une chaire de recherche "Transition 2 : des espaces en transition à la transition des espaces éducatifs » (Jeannin) sur l'architecture de demain fait suite au projet «Archiclasse », un projet pour une architecture scolaire innovante compatible avec le numérique (2016)

4. Dès 2006, une volonté de sanctuariser les écoles françaises est mise en avant avec la circulaire du 16 août sur la lutte contre les violences scolaires. L'un des six objectifs fixé est d'« assurer la sécurité des personnes ». Cela s'illustre par la réalisation d'un diagnostic et un audit de sécurité dans les établissements scolaires connaissant des situations complexes. Il pouvait être complété par un audit de prévention situationnelle établi avec les partenaires locaux (au premier rang desquels les maires et les présidents de conseil général ou régional), faisant apparaittre la liste des recommandations susceptibles d'améliorer la protection et la surveillance des établissements, en particulier en se prémunissant contre les risques d'intrusions. » Suivront une série de circulaires qui verra naître les équipes mobiles de sécurité ou la généralisation des diagnostics de sécurité.

5. Article "Lille : il est comment le nouveau collège ? La parole aux élèves de Moulins ", La Voix du Nord du 11/11/2015, http://www.lavoixdunord.fr/archive/ lille-il-est-comment-le-nouveaucollege-la-parole-aux-ia19b57396n3154551.

6. Une salle pupitre est une salle qui permet à chaque élève de disposer d'un poste informatique. Sur son ordinateur, l'élève a pu se rendre grâce à un lien internet sur l'enquête déposée en ligne et y répondre directement.

7. Rigolon (2010) a identifié quatre types d'architecture scolaire. Au type « cour », s'ajoutent le "bloc» aux volumes compacts, à l'implantation intérieure simple et à son unique espace de socialisation desservant les espaces d'apprentissage, la "grappe » dont «le bâtiment est fragmenté en différents volumes, qui peuvent présenter des unités pédagogiques indépendantes et la « ville » s'illustrant par une multiplicité des espaces et d'unités d'apprentissage.

8. Sur l'échelle de Likert proposée aux répondants, 1 signifiait «pas du tout», $2:$ « un peu », 3 : «moyennement », $4:$ « beaucoup » et $5:$ «énormément ».

\section{RÉSUMÉS}

Partant du postulat que l'environnement peut être perçu comme une menace au bien-être des individus, l'objet de cet article est de proposer à partir d'une enquête quantitative, une évaluation des qualités architecturales de trois récents collèges. Il s'agissait pour des élèves d'exprimer leur ressenti sur leur bâtiment scolaire. Les résultats ont révélé qu'ils le connotent plutôt positivement. Ce ressenti, fortement corrélé aux émotions éveillées par ce lieu, laisse toutefois émergé des facteurs d'influence négative du bien-être (le bruit, l'absence de couleur et de lumière naturelle) et la problématique de l'« école sanctuaire » ou des « espaces de liberté ».

Starting from the premise that the environment can be perceived as a threat to the well-being of individuals, the purpose of this article is to propose, based on a quantitative survey, an assessment of the architectural qualities of three recent colleges. The aim was for students to express their feelings on their school building. The results showed that they are quite positive about it. This feeling, strongly correlated with the emotions aroused by this place, however, leaves emerged factors of negative influence of well-being (noise, lack of colour and natural light) and the problem of the "sanctuary school" or "spaces of freedom". 
INDEX

Mots-clés : architecture scolaire, bien-être, élèves, émotions

Keywords : school architecture, well-being, students, emotions

\section{AUTEURS}

\section{THIBAUT HÉBERT}

ESPE Lille Nord de France, Communauté d'Universités et d'établissements Lille Nord de France, RECIFES EA 4520

\section{ERIC DUGAS}

ESPE d'Aquitaine, Université de Bordeaux, LACES EA 7437 